\title{
Factors affecting the choice of therapeutic regimens in asthma and chronic obstructive pulmonary disease patients and patient adherence to the therapies prescribed
}

\author{
Paweł Nastałek ${ }^{1}$, Adam Kurek², Agnieszka Almgren-Rachtan³ ${ }^{3}$ Agnieszka Mastalerz-Migas ${ }^{4}$, Zbigniew Doniec $^{5}$
}

${ }^{1}$ Pulmonology Clinic, Jagiellonian University Medical College, Krakow, Poland

${ }^{2}$ Department of General Surgery, Vascular and Transplant Surgery, Medical University of Silesia, Katowice, Poland

${ }^{3}$ Department of Pharmacovigilance, Europharma, Katowice, Poland

${ }^{4}$ Department of Family Medicine, Wroclaw Medical University, Wroclaw, Poland

${ }^{5}$ Department of Pneumonology, Institute of Tuberculosis and Lung Diseases, Rabka Branch, Rabka-Zdroj, Poland

Adv Dermatol Allergol 2019; XXXVI (2): 202-210

DOI: https://doi.org/10.5114/ada.2018.76144

\begin{abstract}
Introduction: Chronic obstructive pulmonary disease (COPD) and asthma are the most common chronic respiratory diseases worldwide. At the moment, there is no information about the preferences of Polish specialists as regards the treatment of asthma and COPD or factors influencing the choice of particular treatment regimens.

Aim: To determine the treatment options most commonly used by experienced pulmonologists and allergists for asthma and COPD and to identify the factors affecting the choice of a particular therapy.

Material and methods: The survey included 224 doctors (pulmonologists and allergists) across Poland and concerned patients diagnosed with asthma $(n=4358)$ and COPD $(n=3062)$.

Results: In the case of asthma, the most common therapy applied was inhaled glucocorticosteroids and long-acting $\beta_{2}$ agonists. According to $27.2 \%$ of doctors, combination therapy was used in $70-80 \%$ of patients while $23.7 \%$ declared that the proportion of patients receiving such a treatment exceeded $80 \%$. In the case of COPD, anticholinergics were most frequently prescribed when inhaled glucocorticosteroids and long-acting $\beta_{2}$ agonists had proved insufficient. According to $21 \%$ of specialists, the percentage of patients treated so was $41-50 \%$, while $19 \%$ declared the use of this treatment in $71-80 \%$ of patients.

Conclusions: The most common treatments for asthma and COPD in Poland are inhaled glucocorticosteroids and long-acting $\beta_{2}$ agonists. The main factors influencing treatment decisions are the current GINA and GOLD recommendations as well as patients' age, comorbidities, and price of treatment.
\end{abstract}

Key words: asthma, chronic obstructive pulmonary disease, therapeutic preferences, therapeutic regimens.

\section{Introduction}

Chronic obstructive pulmonary disease (COPD) and asthma are the most common chronic respiratory diseases worldwide [1-3].

Chronic obstructive pulmonary disease represents a serious public healthcare challenge and is one of the major causes of chronic morbidity and mortality throughout the world and is predicted to be the third leading cause of death by 2020 [1, 3]. According to the 2017 GOLD report, COPD is a preventable and treatable disease which is characterized by persistent respiratory symp- toms (dyspnoea and sputum production) and airflow obstruction due to airway and/or alveolar abnormalities usually caused by significant exposure to noxious particles or gases [1]. Tobacco smoking is considered the most important COPD risk factor but other environmental exposures such as biomass fuel exposure and air pollution may also contribute. It is estimated that in Poland around two million people suffer from COPD. Airflow obstruction GOLD $\geq 2$ (moderate degree of obstruction) may be found in approx. $10 \%$ of the Polish population over the age of 40 .

Address for correspondence: Adam Kurek MD, Department of General Surgery, Vascular and Transplant Surgery, Medical University of Silesia, 20-24 Francuska St, 40-027 Katowice, Poland, phone: +48 723993 819, e-mail: adamkurek@outlook.com Received: 16.05.2017, accepted: 5.02.2018. 
Chronic obstructive pulmonary disease assessment must include the severity of airflow obstruction (spirometrically measured), proper symptoms' evaluation (assessed objectively with appropriate questionnaires), obtaining the history and future risk of exacerbation and presence of comorbidities [2, 3]. The latest GOLD guidelines propose a new approach to COPD assessment by separating spirometric grades from the "ABCD" groups [3]. Spirometry still remains the first-line tool to confirm irreversible airflow obstruction but respiratory symptoms and exacerbation history are crucial for therapeutic approaches.

The goal of the COPD therapy is to reduce symptoms as well as the frequency and severity of exacerbations, and improve exercise tolerance and overall health status. The classes of medications commonly used to treat COPD include bronchodilators that increase forced expiratory volume in $1 \mathrm{~s}\left(\mathrm{FEV}_{1}\right)$ and/or change other spirometric variables. They are most often prescribed on a regular basis to prevent or reduce symptoms. The first group of bronchodilators consists of $\beta_{2}$-agonists both short-acting (SABA) and long-acting (LABA) ones. Formoterol and salmeterol are twice-daily LABAs. Indacaterol, olodaterol and vilanterol are additional once-daily LABAs. The second important group of bronchodilators comprises antimuscarinic drugs: short-acting antimuscarinics (SAMAs) such as ipratropium and long-acting antimuscarinic antagonists (LAMAs) including tiotropium, aclidinium, glycopyrronium bromide and umeclidinium. Anti-inflammatory drugs are represented by inhaled glucocorticosteroids (ICS), oral glucocorticosteroids (OCS), phosphodiesterase-4 (PDE4) inhibitors (roflumilast), mucolytics and antibiotics. These are mentioned only when used in combination with bronchodilators. The latest GOLD report also points out to the significance of non-pharmacologic strategies including smoking cessation, education, selfmanagement and pulmonary rehabilitation. Stable COPD pharmacologic treatment regimen proposed by GOLD 2017 guidelines has been summarized in Table 1.

Asthma is a heterogeneous, chronic obstructive inflammatory airway disease affecting up to $18 \%$ of population in different countries. The prevalence of asthma in Poland is estimated at $5 \%$ of the population. It is characterized by symptoms of airflow limitation such as wheeze, shortness of breath or cough which, in contrast to COPD, may vary over time. Asthma may be manifested in various clinical phenotypes that require different clinical approach and management. Diagnosis of asthma is based on the presence of typical symptoms and the evaluation of variable airflow limitation measured mainly by the lung function test and peak expiratory flow rate evaluation. In certain phenotypes of asthma, other clinical tools such as allergy and bronchial provocation tests are useful for diagnosis. Once the diagnosis is established, the level of asthma control should be assessed. It includes the evaluation of certain symptoms' frequency: daytime symptoms, nocturnal awakenings due to asthma, need for relief medication and limitation of physical activity. On the basis of these criteria and in accordance with the current GINA guidelines, asthma is classified as controlled, partly controlled, and uncontrolled.

The treatment regimen depends not only on the level of asthma control but it is also shaped by severity of airflow limitation, risk of exacerbation, presence of comorbidities, patient preferences and ability to use different types of inhalers. The goal of the therapy is to achieve the best available control of the symptoms, to improve the quality of life and to minimize the risk of disease exacerbation. The current (2107) GINA guidelines propose a control-based asthma management protocol and point out to other asthma treatment strategies that may play an important role in severe or difficult to treat asthma e.g. sputum-guided treatment or fractional concentration of exhaled nitric dioxide (FeNO)-guided regimen. Inhalation therapy is the principal therapeutic modality in all asthma phenotypes. Asthma medications can be divided into three main groups:

- Controller medications - reduce airway inflammation, patient symptoms and risk of asthma exacerbation. These include ICS (beclomethasone, budesonide, ciclesonide, fluticasone and mometasone), antileukotriene drugs (montelukast, zafirlukast), LABA (formoterol, salmeterol), tiotropium and theophylline.

- Relievers - include SABA (fenoterol, salbutamol) and combination of low-dose ICS and formoterol in one inhaler.

- Add-on therapies which should be considered in the cases of all patients with poor response to treatment (e.g. tiotropium and omalizumab).

Table 1. GOLD 2017 recommendations for stable chronic obstructive pulmonary disease treatment

\begin{tabular}{cc}
\hline C & D \\
LAMA & \\
or & LAMA + LABA \\
or \\
LAMA + LABA & LAMA + LABA + ICS \\
or & (if further exacerbations occur) \\
LABA + ICS & or \\
(if further exacerbations & LAMA \\
occur) & or \\
& LABA + ICS \\
& +roflumilast (FEV $1_{1}<50 \%$ \\
and chronic bronchitis)
\end{tabular}

LABA - long-acting $\beta_{2}$-agonists, LAMA - e.g. long-acting anticholinergics, ICS - inhaled glucocorticosteroids. 
Table 2. Asthma pharmacological treatment stepwise approach according to GINA 2017

\begin{tabular}{|c|c|c|c|}
\hline $\begin{array}{l}\text { Intensity levels of asthma } \\
\text { treatment }\end{array}$ & Preferred controller choice & Other controller option & Reliever \\
\hline Step 1 & \multirow[t]{2}{*}{ Low-dose ICS } & Consider low-dose ICS & \multirow[t]{2}{*}{ As-needed SABA } \\
\hline Step 2 & & $\begin{array}{c}\text { LTRA } \\
\text { Low-dose theophylline }\end{array}$ & \\
\hline Step 3 & Low-dose ICS/LABA & $\begin{array}{c}\text { Medium/high-dose } \\
\text { ICS/LABA } \\
\text { Low-dose ICS + LTRA } \\
\text { (or + theophylline) }\end{array}$ & \multirow[t]{3}{*}{$\begin{array}{l}\text { As-needed SABA or } \\
\text { low-dose ICS/formotero }\end{array}$} \\
\hline Step 4 & $\begin{array}{l}\text { Medium/high-dose } \\
\text { ICS/LABA }\end{array}$ & $\begin{array}{l}\text { Add tiotropium } \\
\text { High-dose ICS + LTRA } \\
\text { (or + theophylline) }\end{array}$ & \\
\hline Step 5 & $\begin{array}{l}\text { Refer for add-on treatment } \\
\text { (e.g. tiotropium, omalizumab) }\end{array}$ & Add low-dose OCS & \\
\hline
\end{tabular}

ICS - inhaled glucocorticosteroids, SABA - short-acting $\beta_{2}$-agonists (e.g. fenoterol and salbutamol), LABA - long-acting $\beta_{2}$-agonists (e.g. formoterol and salmeterol), OCS - oral glucocorticosteroids.

The 2017 GINA guidelines recommend a stepwise pharmacologic treatment regimen for asthma which are summarized in Table 2. The document also indicates importance of non-pharmacologic interventions in asthma. These mainly include guided self-management education and several strategies e.g. weight loss, increased physical activity, and avoidance of sensitizers and proper management of comorbidities.

\section{Aim}

The aim of this study was to determine the asthma and COPD treatment regimens most commonly used by experienced pulmonologists and allergists and to identify the factors affecting the choice of a particular therapy. The data obtained in this paper may help to identify the patterns applied in the therapeutic decision-making process as well as patient adherence issues.

\section{Material and methods}

The survey was conducted across Poland in the first half of 2016 and was concerned with patients diagnosed with asthma and COPD. The doctors received a questionnaire which they completed and returned to the research centre. The questionnaire consisted of two parts. Part A contained questions concerning statistical data referring to the doctors: how long the doctor worked in the profession, place of work (city/village and public/private hospital or public/private clinic), the number of patients seen per month, the percentage of patients diagnosed with asthma and COPD in a month, and the percentage of patients who used the listed groups of drugs (alone or in combination). Part B consisted of questions concerning patient characteristics including demographics, education and occupation, place of residence (urban/rural), initial diagnosis at baseline, disease duration, the degree of disease control, the number of exacerbations, and hospitalizations in the past 3 months and prescribed treatment regimens.

Based on the questionnaires we conducted an analysis of the doctors' (allergists and pulmonologists specializing in the treatment of asthma and (OPD) and patients' preferences depending on the doctors' length of service, patients' age and professional activity, treatment duration and occurrence of comorbidities.

\section{Results}

A total of 224 doctors responded to the questionnaire. Out of these, $78 \%$ were pulmonologists, $21 \%$ allergists; $69 \%$ had over 20 years of professional experience. The doctors were mainly employed by different clinics: $54.9 \%$ - public and $15.6 \%$ - private, while hospital was the principal place of work for $21.9 \%$ of doctors. The majority of doctors (90.6\%) worked in cities. Each saw an average of 400 patients per month. The number of patients they saw with asthma or COPD in most cases ranged from 20 to 50 per month.

The survey was conducted on a group of $7420 \mathrm{pa}-$ tients (4009 males; 54\%) out of whom 4358 had been diagnosed with asthma and the remaining 3062 with COPD. The average patient age was $50.6 \pm 16.9$ years. Of the respondents, $23.5 \%$ were rural residents, $61.4 \%$ of patients were still working. In a majority of respondents, the illness had lasted for over 5 years. During the 3 months prior to the survey, $27.43 \%$ of patients with asthma and $48 \%$ patients with COPD experienced at least one exacerbation. In the 3 months prior to the study, $4.4 \%$ of patients with asthma required hospitalization due to exacerbation. In patients with COPD, the hospitalization percentage was $23.3 \%$. Asthma was rated as controlled in $55 \%$ of patients. Out of the total of patients with COPD, $65.9 \%$ were classified as GOLD category C and D. Detailed medical records are included in Table 3. 
Table 3. Data from patient interview

\begin{tabular}{lcc}
\hline Parameter & Asthma $(n=4358)$ & COPD $(n=3062)$ \\
\hline \begin{tabular}{l} 
Duration of the disease, $n(\%):$ \\
\hline$<3$ months
\end{tabular} & $113(2.6)$ & $26(0.8)$ \\
\hline 3-6 months & $104(2.4)$ & $25(0.8)$ \\
\hline 7-12 months & $170(3.9)$ & $67(2.2)$ \\
\hline 1-2 years & $326(7.5)$ & $148(4.8)$ \\
\hline 2-3 years & $763(17.5)$ & $594(19.4)$ \\
\hline 4-5 years & $823(18.9)$ & $626(20.4)$ \\
\hline$>5$ years & $2059(47.2)$ & $1576(51.5)$ \\
\hline Exacerbation of the disease in the last 3 months, $n(\%)$ & $1196(27.4)$ & $1470(48.0)$ \\
\hline If so, how many? Mean \pm SD & $1.3 \pm 0.6$ & $1.4 \pm 0.6$ \\
\hline Hospitalization due to worsening in the last 3 months, $n(\%)$ & $193(4.4)$ & $714(23.3)$ \\
\hline If so, how many? Mean \pm SD & $1.1 \pm 0.3$ & $1.1 \pm 0.3$ \\
\hline Severity, $n$ (\%): & & $261(8.5)$ \\
\hline Asthma controlled/Category A & $2395(55.0)$ & $782(25.5)$ \\
\hline Asthma partly controlled/Category B & $1661(38.1)$ & $1231(40.2)$ \\
\hline Asthma uncontrolled/Category C & $302(6.9)$ & $788(25.7)$ \\
\hline Category D & & \\
\hline
\end{tabular}

The majority of doctors surveyed declared that their most common (49.5\%) therapeutic decision in patients with asthma was the prescription of ICS and LABA, and $27.2 \%$ declared that they prescribed combination therapy in $70-80 \%$ of patients. According to $23.7 \%$ of doctors, the percentage of patients receiving such treatment exceeded $80 \%$. Other frequently used treatment options were also short-acting $\beta_{2}$ mimetics and ICS. Theophylline was rarely used in these patients. The key factors to influence the therapeutic decisions were the GINA guidelines (66.5\% of respondents). Other factors strongly affecting treatment decisions were etiological ones associated with the development of the disease, comorbidities, the price of the drug and patient preference.

A detailed overview of therapeutic decisions in patients with diagnosed asthma is presented in Table 4.

For patients with COPD, the most common therapeutic approach has been the inclusion of an anticholinergic drug, followed, as in the case of asthma, by ICS and LABA. The doctors also often declared prescribing SABA. According to $21 \%$ of doctors, the percentage of patients receiving combination therapy with ICS and LABA ranged from $41 \%$ to $50 \%$. The above scheme was applied by $19 \%$ of specialists in $71-80 \%$ of patients and only $6.3 \%$ declared using it in more than $80 \%$ of patients. The key factor influencing treatment decisions were the GOLD recommendations (65\% of respondents). Quality of life, morbidity, and co-occurrence of asthma also played an important role in the treatment selection process. A detailed presentation of therapeutic decisions in patients diagnosed with COPD is featured in Table 5.
In their treatment of patients with asthma, both pulmonologists and allergists were willing to prescribe combination treatment with ICS and LABA. Allergists were much more likely to select SABA. As far as other groups of drugs were concerned, the specialists acted quite similarly taking their decisions mostly in accordance with the GINA guidelines, the etiologic factors of the disease, quality of life, and concomitant diseases. Allergists, more often than pulmonologists, took account of other factors such as the price of the drug and patients' preferences. The differences in treatment preferences were less noticeable in patients with COPD. Allergists often declared the use of combined treatment with ICS and LABA in this group of patients and prescribed drugs from other groups with similar frequency as the pulmonologists. There were no significant differences between the specialists in terms of the factors influencing the choice of a particular therapeutic regimen. A detailed overview of therapeutic decisions in patients with diagnosed asthma and COPD are presented in Tables 4 and 5.

The doctors' length of service did not significantly influence their therapeutic decisions in patients with asthma as these were usually based on the GINA guidelines. In patients with COPD, specialists with a shorter length of service often opted for a particular therapy taking into account patient age and asthma comorbidity. For doctors with longer professional experience, more important factors were the price, tolerability of the drug, and patients' personal preferences. The GOLD guidelines however were the most important factor for all the surveyed doctors during their therapeutic decision-making process. 


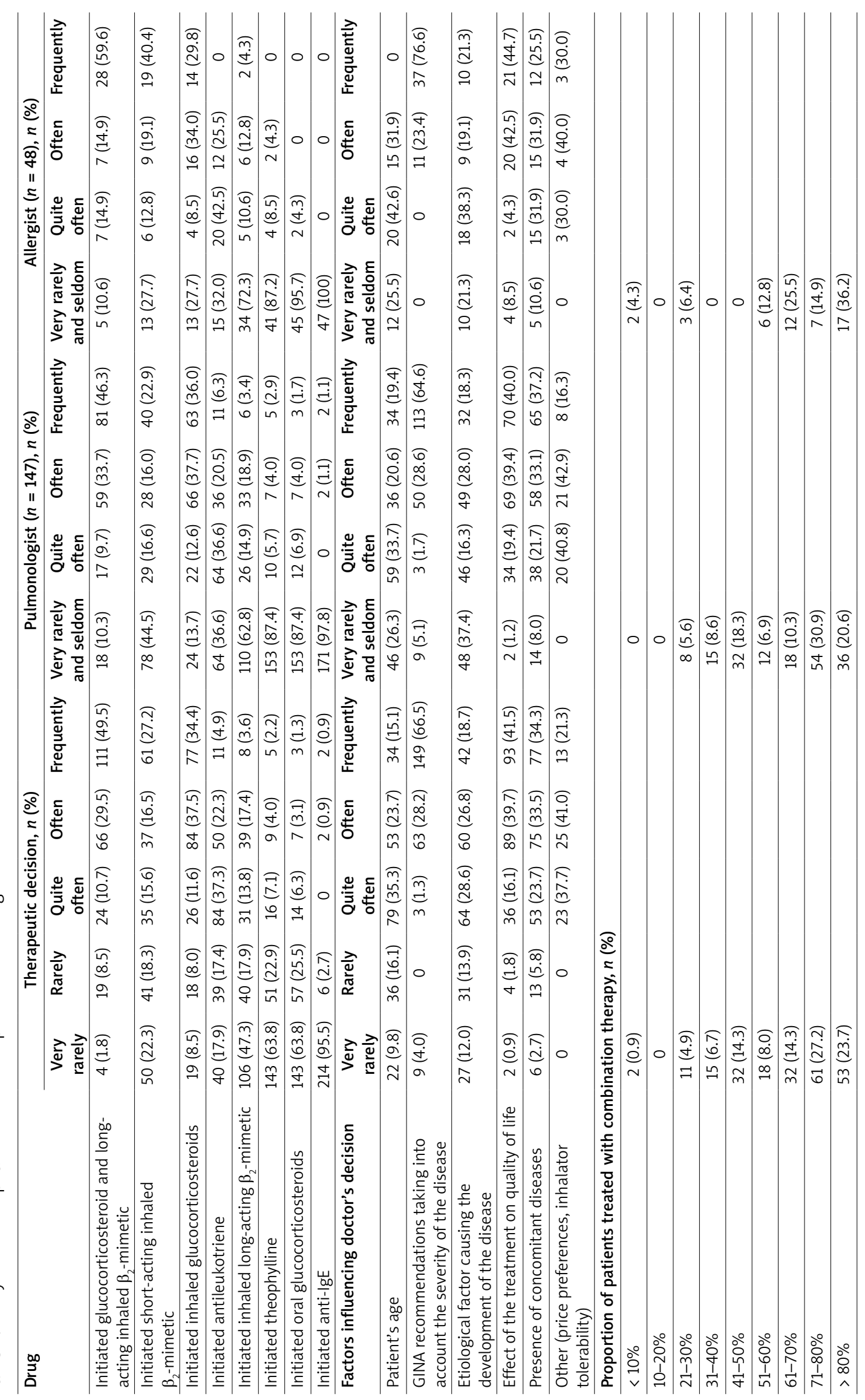




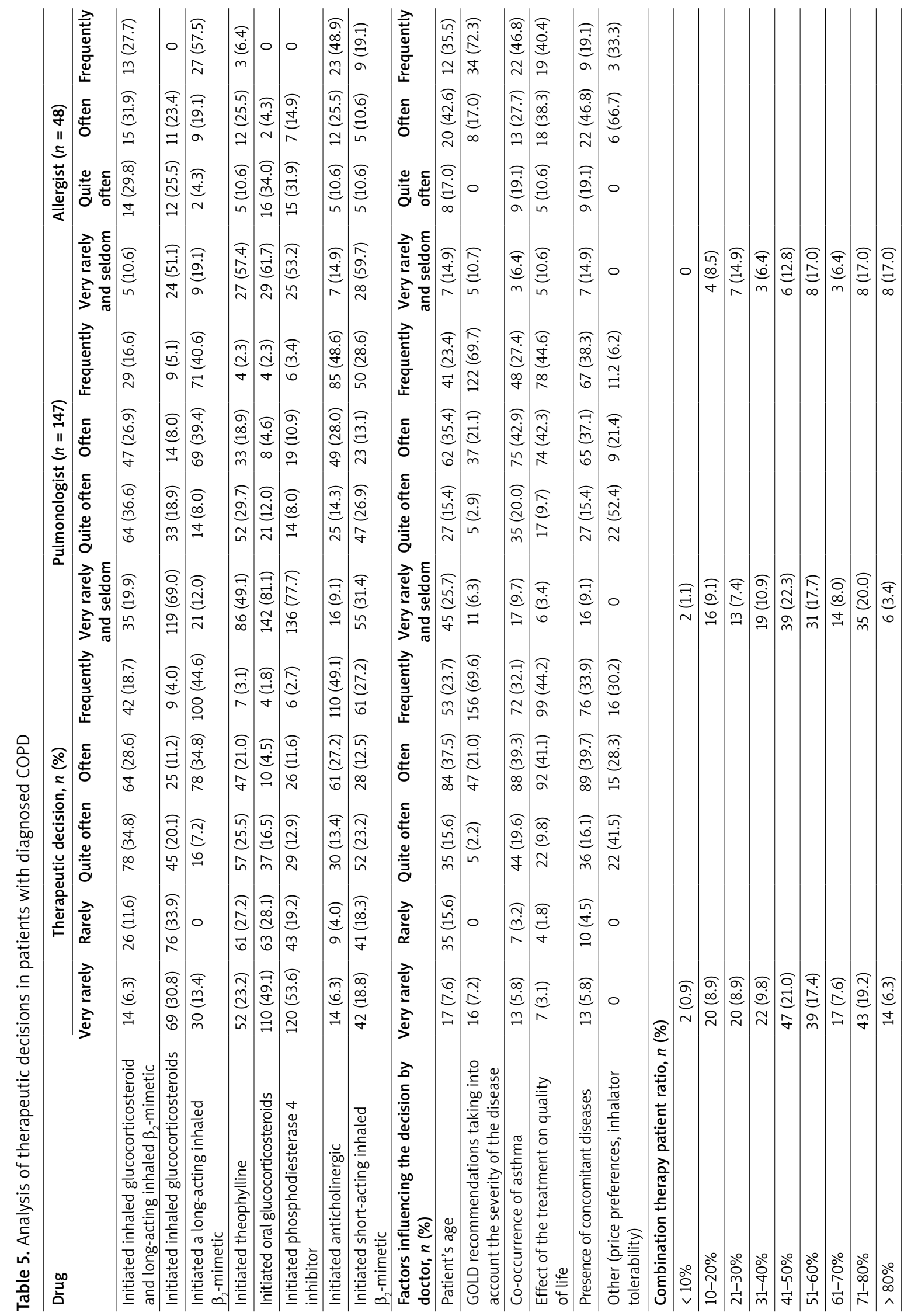


Table 6. Treatment applied

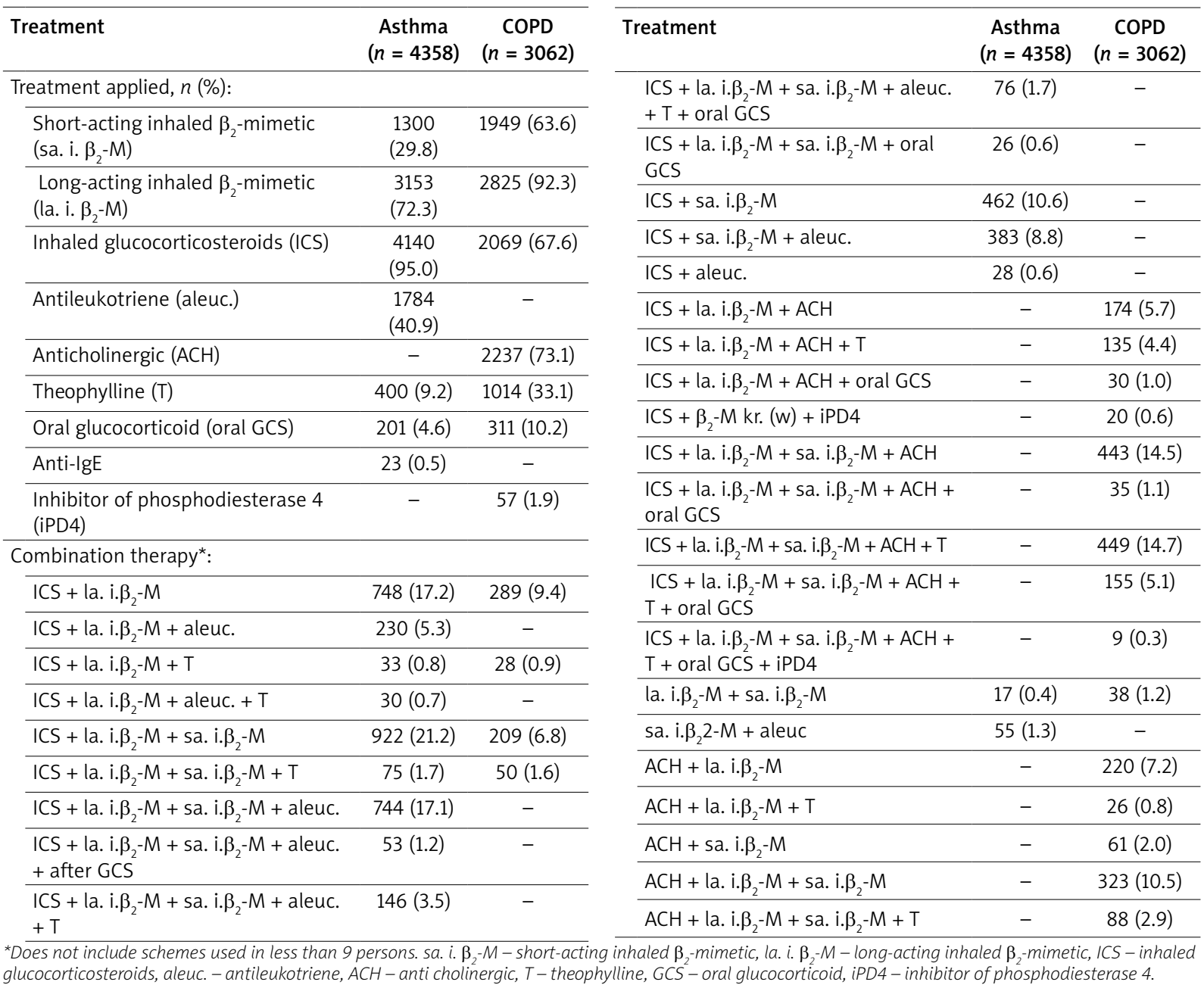

Regardless of their workplace, the doctors in the survey prescribed combination therapy with ICS and LABA in patients with asthma. Compared with hospital treatment, outpatient clinics more often used inhaled GCS, short-acting $\beta_{2}$-adrenergic agonists and antileukotrienes. The doctors working at hospitals more often took into account their patients' age and etiological factors of the disease. In outpatient clinics, emphasis was placed on the preferences of the patient and the price of the drug. Most often and independently of the location, therapeutic decisions were based on the GINA guidelines. In the hospital patients with COPD, anticholinergics were frequently used. The factors that influenced therapeutic decisions in hospitals and outpatient clinics were similar, the most important being the GOLD guidelines. In the outpatient clinics, patients' preferences and the price of drug were more important just like in the case of the patients with asthma. The most commonly used therapeutic regimens for asthma and COPD depending on the specialist are presented in Table 6.
Analysis was also conducted in reference to the patient-doctor collaboration in the treatment of asthma and COPD. It was estimated that in the case of asthma, medical recommendations were applied by $91.0 \%$ of men and $92.2 \%$ of women while in COPD these ratios were $85.7 \%$ and $88.4 \%$, respectively. Significantly lower levels of adherence to medical recommendations were found in COPD patients, especially younger ones ( $\geq 40$ years). Only $68.3 \%$ of COPD patients adhered to their doctors' recommendations (vs. $90.9 \%$ in the case of asthma). The level of education was also another important therapy adherence predictor in COPD patients as only $81.5 \%$ of patients with vocational training complied with the recommendations of their doctors (vs. 95.2\% of COPD patients with higher education).

\section{Discussion}

Patients with obstructive lung diseases are usually seeking advice in both pulmonary and allergy clinics. In the Polish healthcare system, pharmacotherapy pre- 
scribed by specialists in asthma and COPD is a crucial factor determining the long-term treatment of these patients. This study, conducted with the participation of experienced professionals on a large group of patients, is an attempt to answer the questions of how the treatment of asthma and COPD is carried out by pulmonologists and allergists in their everyday clinical practice as well as what factors influence the choice of a particular therapy.

Pulmonologists and allergists should first pay attention to the population of patients participating in the study. Most patients with COPD were classified as category $C$ and $D$ and among patients with asthma only just over a half were characterized by a good degree of control. Most patients had been suffering from asthma or COPD for many years. Over $25 \%$ of the patients with asthma and $50 \%$ of those suffering from COPD had experienced exacerbation of the disease within 3 months prior to the study. In many cases they required hospitalization. These findings certainly should be taken into account when selecting pharmacotherapy.

The preferred therapeutic option for pulmonologists and allergists was to prescribe a combination of inhaled corticosteroids and long-acting $\beta_{2}$-adrenergic agonists, both to patients with asthma and COPD. Frequent use of such a combination therapy for asthma is fully understood and both agents are in fact the basis of pharmacotherapy in these diseases, particularly if control is insufficient. The benefits of combination therapy have been confirmed by the results of the patients included in this study. In this context it is not surprising that (more frequently than in COPD) ICS with $\beta_{2}$-adrenergic agonists are recommended by the GINA especially in milder forms of asthma as one of the main therapeutic options. The widespread use of combination therapy with inhaled corticosteroids and long-acting $\beta_{2}$-adrenergic agonists may however raise some doubts in the case of COPD patients. While bronchodilators are the initial recommended treatment, indications for ICS are limited. The ICS are prescribed in COPD categories C and D, in patients with frequent exacerbations, and are always combined with bronchodilators. The widespread application of combination therapy consisting of inhaled corticosteroids and LABA reported in this study could be associated with the group of patients included in the study. Most COPD patients were enrolled for $C$ and D GOLD categories, nearly half had experienced exacerbation of the disease and hospitalization was required in every fourth patient. Undoubtedly, the frequent use of anticholinergic drugs in COPD patients is a positive phenomenon. Research shows that their therapeutic efficacy (long-acting anticholinergics) is comparable to treatment with an inhaled corticosteroid and long-acting $\beta_{2}$-adrenergic agonists, with a lower risk of adverse reactions (particularly pneumonia) associated with the intake of inhaled glucocorticoids. Tiotropium, a long-acting mus- carinic antagonist, also has an established place in the GINA guidelines since it is prescribed for severe asthma ( $4^{\text {th }}$ step of therapy) but in the Polish health care system its use in asthma is still insignificant as it is expensive and not refunded. Another positive phenomenon is less frequent use of theophylline in COPD patients, especially in these with asthma. This drug has rather low therapeutic effectiveness and involves a considerable risk of serious side effects.

Patients' compliance with recommendations is a significant clinical issue which cannot be overlooked in the treatment of asthma and COPD and the most important factor in obtaining adequate control of the disease and prevent exacerbations as well as potential hospitalizations.

Reviewing the latest literature in the field of obstructive lung diseases, adherence to therapy in asthma and COPD was a frequent subject of research [4-8]. The results of the present study confirm that the GINA and GOLD guidelines are the essential factors determining the specialists' choice of therapy regimen. Noteworthy is the fact that both pulmonologists and allergists take into account not only guidelines but also other crucial factors that may affect adherence to therapy. In clinical practice, the factor that often turns out to be the key one for poor adherence is patients' age [4, 5]. Advanced age is frequently associated with cognitive impairment. In O'Conor's study [4], 27\% of asthmatic patient population (mean age of the sample was 67 years) were classified as high cognitive impairment according to the minimental status examination score. Authors emphasized that decreased cognitive function may be associated not only with a poor inhalation technique but may also affect other parts of the disease treatment plan i.e. using a different type of medications (maintenance vs. emergency), recognizing exacerbations and general healthcare selfmanagement. Cognitive dysfunction may also play an important role in comorbidity that strongly affects not only adherence to therapy but overall treatment outcomes as well. In Turan study [5], mistakes in the inhalation technique in elderly both asthmatic and COPD patients were present in $90 \%$ of individuals and were more frequent in COPD patients. The results of the present study confirm observation of poor adherence in COPD patients, what seems to be a serious clinical problem in all chronic respiratory diseases with hypoxemia. Cognitive dysfunction is frequently associated with a low socioeconomic status, another poor therapy adherence risk factor [4-6]. In the present study, the problem of non-compliance with the doctor's instructions was a frequent finding in less educated COPD and asthmatic patients. The socioeconomic status in the Polish healthcare system plays an additional important role because of great reimbursement differences for certain therapy agents. In the present study, the agent price proved to be one of the key ones in terms of patients' treatment compliance, being clearly more often 
recognised by more experienced professionals. In contrast to this observation, Voorham et al. [6] did not prove that prescription charges impact therapy adherence and diseases outcomes. It can be explained by differences in the healthcare system and general higher economic status in Western Europe countries comparing to Poland. The present study also indicates the importance of patient preferences for a particular product and the type of inhaler. This observation is supported by the Dutch research [7]. Authors concluded that adherence to inhalation therapy for COPD treatment is strongly related to the medication prescribed, pointing out tiotropium as an agent with the best level of adherence. It can be explained not only by efficacy of this agent but probably by the dosing regimen (once a day) and the inhaler type. Referencing this observation to Polish COPD population, the high frequency of using combined inhalers may also have a purpose of improving compliance. An asthma adherence determinant was the subject of a separate systematic review [8]. Findings suggest that adults with asthma better adhere to therapy if they are well informed and better educated about the disease and are less concerned about using inhalers. However, younger adult patients may be more at risk of nonadherence. This observation is consistent with the present study.

\section{Conclusions}

The results of this study suggest further that the overall degree of Polish patient compliance is high. This observation may however raise some doubts, especially in the context of the prevalence of exacerbations and insufficient disease control, pointing to the need for continuous education of both patients and physicians.

\section{Acknowledgments}

The study was carried out as a research project supported by a scientific grant from Polfarmex S.A. contracted by Europharma. Participating specialists received payment for completing the questionnaires.

\section{Conflict of interest}

The authors declare no conflict of interest.

\section{References}

1. GOLD Global Initiative for Obstructive Lung Disease - Global Strategy for The Diagnosis, Management and Prevention of Chronic Obstructive Pulmonary Disease. Report 2017 goldcopd.org

2. Global Initiative for Asthma (GINA): Global Strategy for Asthma Management and Prevention. WHO/NHLBI Workshop Report 2017. ginasthma.org

3. Śliwiński P, Górecka D, Jassem E, et al. Zalecenia Polskiego Towarzystwa Chorób Płuc dotyczące rozpoznawania i lecze- nia przewlekłej obturacyjnej choroby płuc (POChP). Pneumonol Alergol Pol 2014; 82: 227-63.

4. O'Conor R, Wolf MS, Smith SG, et al. Health literacy, cognitive function, proper use, and adherence to inhaled asthma controller medications among older adults with asthma. Chest 2015; 147: 1307-15.

5. Turan O, Turan PA, Mirici A. Parameters affecting inhalation therapy adherence in elderly patients with chronic obstructive lung disease and asthma. Geriatr Gerontol Int 2017; 17: 999-1005.

6. Voorham J, Vrijens B, van Boven JF, et al. Does co-payment for inhaler devices affect therapy adherence and disease outcomes? A historical, matched cohort study. Pragmat Obs Res 2017; 8: 31-41.

7. Huurne KK, Movig K, van der Valk P, et al. Differences in adherence to common inhaled medications in COPD. COPD 2015; 12: 643-8.

8. Dima A, Hernandez G, Cunillera O Asthma inhaler adherence determinants in adults: systematic review of observational data. Eur Respir J 2015; 45: 994-1018. 